\title{
comprobación experimental de cubiertas laminares, por medio de modelos reducidos
}

\section{BENITO}

\author{
Ingeniero de Caminos Canales y Puertos
}

Encargado de la Sección de Modelos Reducidos de Estructuras del Laboratorio Central

de Ensayo de Materiales de Construcción

"The problem of covering large areas using the minimun of intermediate supporting nembers is one which has appealed to architects and engineers for centuries..."

$$
\text { E. D. MILLS }
$$

\section{1. introducción}

La sensación de grandiosidad, la admiración que produce al espectador la contemplación de una gran sala o recinto cubierto y totalmente exento de soportes o columnas intermedias, ha sido buscada por constructores de todos los tiempos. A este efecto, se ha sumado la perfecta visibilidad ofrecida por tales recintos, útil siempre para reuniones y, actualmente, por la libertad que ofrece para cambiar los planes de distribución de espacios; cambios exigidos en las grandes fábricas, por los continuos avances de la técnica industrial.

Al no contar los constructores, hasta fecha reciente, con materiales que poseyesen una elevada resistencia a tracción-problema que se resuelve con la llegada del acero, y, mejor aún, del hormigón armado-y conocedores, sin embargo, de dos formas resistentes, la viga y el arco, es lógico pensar que los constructores se hayan visto impelidos a utilizar este último en forma de bóvedas y cúpulas, como más idóneo a las características de los materiales por ellos empleados. La cúpula del Panteón, con sus 42 me- 
tros de diámetro, las bóvedas en cañón de muchos edificios públicos, que construídos por la Roma imperial se han conservado hasta nuestros días, así lo atestiguan.

La necesidad, quizás presentida por los técnicos antiguos, de centrar en lo posible la línea de presiones en las diversas secciones de la cubierta, para que los materiales trabajasen a compresión, les llevó a espesores considerables que, al aumentar el peso propio, dificultaban aún más el problema resistente. Aunque, por otra parte, procurasen disminuir los pesos, embebiendo en la masa recipientes construídos con materiales cerámicos.

Estas cubiertas se solían apoyar por su contorno sobre muros corridos de gran espesor, necesarios para absorber, en buenas condiciones de equilibrio, los empujes inclinados que son consecuencia obligada de las formas curvas. La intuición de los constructores y algún que otro desastre, les enseñaría a contrarrestar empujes entre bóvedas contiguas o de cúpulas, bóvedas y ábsides como en la catedral de Santa Sofía, resultado feliz de este tipo de transmisión de empujes, en donde con una cúpula de $30 \mathrm{~m}$ de diámetro, arcos y medias cúpulas hábilmente enlazadas se ha logrado una planta de $30 \times 67 \mathrm{~m}$ sin un solo soporte intermedio.

El encuentro de bóvedas por arista y en rincón de claustro permite concentrar los empujes en puntos aislados, lo cual fué aprovechado por los maestros del gótico, quienes, manejando diestramente los arbotantes y botareles, pudieron alcanzar una maravillosa sensación de ingravidez en sus catedrales.

Mucho podría relatarse aquí sobre la influencia de los materiales en las formas resistentes, y de cómo aquéllos se rebelaron algunas veces contra el hombre. Sabemos que después de terminada la cúpula de San Pedro, fué necesario añadir varios anillos de hierro para que absorbieran las tracciones en paralelos inferiores.

Conocemos también el caso de otra cúpula que hubo de construir y reconstruir tres veces, hasta lograr una forma, unos espesores y una sustentación que estuviesen de acuerdo con los materiales utilizados y los pesos por soportar. Los fracasos cuyas referencias han llegado hasta nosotros y algunos que, probablemente, quedarán ignorados, guiaron a nuestros mayores en la concepción de obras maestras y sirvieron para formar el clima propicio al desarrollo de las actuales teorías.

La utilización del hormigón armado en cubiertas de este tipo, tenía forzosamente que revolucionar su concepción. Aportaba lo que les estaba faltando a los materiales hasta entonces empleados: una considerable resistencia a tracción. Ya no importaba que las secciones de la cubierta monolítica trabajasen a flexión simple o compuesta. Se obtenía una mayor libertad en las formas $y$, sobre todo, se alcanzaba una ventaja de primera mgnitud: podían reducirse espesores. Como la carga por soportar en una cubierta era en su casi totalidad el peso propio, éste disminuía, lo que, a su vez, hacía menores las cargas de trabajo, otra vez el espesor, y de nuevo el peso de la cubierta. 

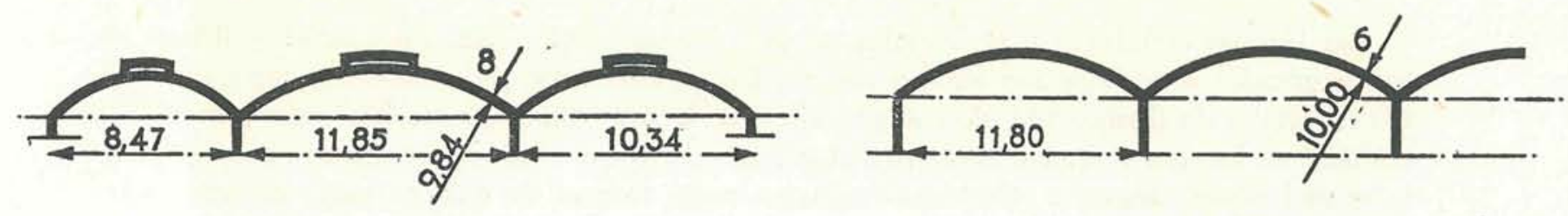

Jena. $23,20 \mathrm{~m}$.

Budapest. $40,00 \mathrm{~m}$.

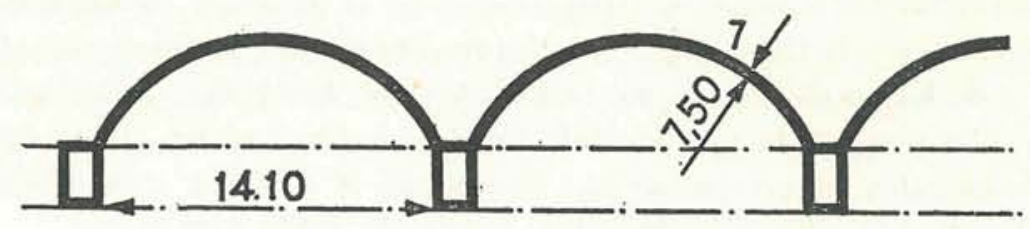

Frankfurt Merk, $36,90 \mathrm{~m}$.

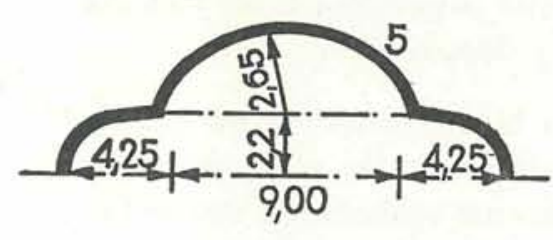

Villaverde $25,00 \mathrm{~m}$.

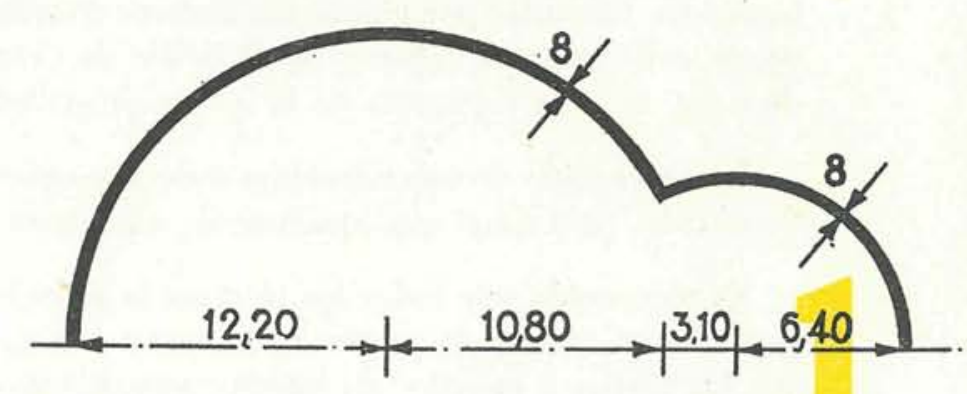

Frontón Madrid 55,00 m.

Con esto empezaba para el proyectista una carrera de reducción de espesores que sólo se vería frenada, en unos casos, por el riesgo de inestabilidad elástica del conjunto, por pandeos locales, o porque, al disminuir la importancia de las cargas por peso propio cobraron realce los efectos atmosféricos (empujes de viento o nieve y variaciones de temperatura). Así se ha llegado al tipo actual de cubierta laminar de hormigón armado, en la que el espesor, como en la lámina de papel, es muy pequeño comparado con el de sus otras dimensiones.

No es de extrañar que las primeras cubiertas laminares tuvieran forma de cúpula, o de bóveda cilíndrica aislada o acompañada. Y así, con láminas cilíndricas de hormigón armado fueron cubiertos, entre otros, el Planetario de Jena (1924), el Mercado de Frankfurt-am-Main (1927), el Mercado de Budapest (1931), la Iglesia de Villaverde (Madrid, 1935) y el Frontón Recoletos de Madrid (1935). En unos casos, la cubierta estaba formada por una sola superficie cilíndrica; mientras en otros, eran varios cilindros los que se unían por alguna de sus generatrices dando origen a secciones con las formas y dimensiones indicadas en la figura 1.

En alguna de las soluciones, las generatrices de borde sirven de apoyo continuo; en otras, los apoyos están constituídos por los dos planos que contienen las directrices extremas. De esta manera, la cubierta, que en su origen tuvo un cercano parentesco con el arco, ha terminado con una forma de trabajar análoga a la de una viga.

Las cubiertas de los mercados de Algeciras ( $47 \mathrm{~m}$ de diámetro), Basilea (52 m), Leipzig (75 m), y la proyectada para el Palacio de los Deportes de Estocolmo $(150 \mathrm{~m})$, son magníficos ejemplos de cúpulas laminares de hormigón armado. Para dar una idea de la disminución alcanzada en el peso propio de la cubierta, podemos indicar que la cúpula de la iglesia de San Pedro, de Roma, con $42 \mathrm{~m}$ de diámetro, pesa cerca de $7 \mathrm{tons} / \mathrm{m}^{2}$, mientras que la del Mercado de Algeciras, algo mayor de diámetro, no llega a los $260 \mathrm{~kg} / \mathrm{m}^{2}$. O sea, 27 veces menos. 
Las láminas cilíndricas y las cúpulas parecen formas ideales para resolver el problema de cubrir grandes superficies con plantas rectangulares o circulares; pero cuando se crean condiciones especiales de iluminación, de sustentación o de forma, originadas muchas veces por motivos estéticos en los que se quiere hacer partícipe a la cubierta, el técnico proyectista, a poco que deje volar su fantasía, llegará a soluciones originales, cuyas formas de trabajar serán distintas a las bóvedas y cúpulas clásicas.

Las cubiertas de las tribunas del Hipódromo de la Zarzuela en Madrid (fig. 2), de la Brymawr Rubber Factory de Gales (fig. 3), de los Auditorios del Massachusetts Institute of Technology (fig. 4) o de Karlsruhe (fig. 5), de la Estación del Aeropuerto Municipal de Lambert, Saint Louis (fig. 6), o la proyectada para el Club Táchira, de Caracas (fig. 7), son unas cuantas muestras de cubiertas laminares, en las que se han encontrado la solución a los problemas funcionales y estéticos sin miedo a la utilización de formas nuevas de doble curvatura.

No quedaría completa esta visión de conjunto, si no citáramos un nuevo grupo de estructuras laminares, formadas por placas triangulares empotradas entre sí elásticamente. A este tipo pertenecen, entre otras, la cubierta del Pabellón de Cristal del Centro Nacional de Deportes, de Londres (fig. 9), y la estructura de la iglesia proyectada por el arquitecto A. Lamela (fig. 8).

La concepción de una estructura o de una cubierta no puede estar supeditada a los métodos de cálculo, pero tiene que ayudarse de ellos para fijar sus formas y dimensiones.

Es reconocida por todos los técnicos la dificultad que presenta la utilización de un método general que sirva para proyectar cubiertas laminares de forma cualquiera. De sobra es sabido que las teorías o métodos de cálculo, actualmente en uso, son solamente aplicables a ciertas formas particulares, tales como láminas cilíndricas, paraboloides, conoides, etc., que han sido sancionadas por la experiencia dentro de unas dimensiones en planta determinadas. Dentro de estos casos especiales, algunas teorías admiten que el material de la lámina es homogéneo y elástico; lo que sólo está de acuerdo con la realidad de manera aproximada, ya que este tipo de cubiertas se construye en la mayoría de los casos con hormigón armado. En las referidas teorías es necesario desarrollar el cálculo de acuerdo con la hipótesis de elasticidad y admitir nuevas simplificaciones, que consisten, por ejemplo, en suponer igual a cero el coeficiente de Poisson del material que constituye la cubierta, y en otros casos que ciertos esfuerzos son nulos o iguales.

Dentro de las láminas cilíndricas, y después de introducir unas condiciones de contorno, se llega, frecuentemente, a una educación diferencial de octavo grado que refleja el equilibrio. En algunos casos es posible resolverla, con lo que se llegan a conocer los esfuerzos que actúan en diversas secciones de la cubierta y así dimensionarlas de acuerdo con las teorías del hormigón armado o del hormigón pretensado.

Cuando la forma de la lámina es de doble curvatura, si no se admite la «hipótesis membrana», no siempre admisible, el cálculo resulta muy difícil, especialmente cuando no es de revolución o cuando las condiciones de borde son complicadas. Es necesario, entonces, recurrir a nuevas simplificaciones de las teorías, con lo que se llega a resultados en los que la correspondencia con la realidad es bastante difícil de evaluar.

De lo expuesto se deduce la necesidad de resolver dos problemas, de verdadera importancia para el proyecto de cubiertas laminares:

1. Aceptadas como válidas unas determinadas hipótesis para desarrollar una teoría o método de cálculo, sería muy conveniente conocer el comportamiento de una o varias cubiertas proyectadas con ellas, con objeto de compararlas con los resultados teóricos, y así, contrastar el grado de precisión del método utilizado.

2. Cuando se dimensiona una cubierta por procedimientos aproximados no sancionados por la experiencia, antes de construirla es necesario prever con suficiente precisión los efectos de las cargas que ha de soportar. 
La auscultación de las construcciones terminadas, puede ser una magnífica fuente de información para resolver el primer problema.

En relación con el segundo, hay que reconocer que los resultados de la auscultación, aunque muy interesantes, son la comprobación de lo inevitable: la obra se cae o no; pero no son útiles al proyectista para la previsión del comportamiento de la misma.

Como se comprobará a lo largo del presente trabajo, el ensayo en modelo reducido de cubiertas laminares suministra resultados satisfactorios para los dos problemas planteados. Ofrécese al técnico la oportunidad de estudiar el comportamiento resistente de una obra de análogas características a la por él ideada, y cuyos resultados son, en todo, semejantes a los que se obtendrían en la obra real.

Aunque ligados entre sí ambos problemas, hemos preferido enunciarles por separado, pues cada uno de ellos requiere una técnica de ensayo distinta.

\section{Ensayos con modelos reducidos}

En general, el modelo reducido de una estructura es una reproducción a escala, de la forma y dimensiones de la misma, construída con materiales apropiados, para que, cargada convenientemente, reproduzca unos fenómenos resistentes y nos proporcione unos resulta. dos cuya naturaleza o magnitud desconocíamos. Por tanto, para realizar un estudio con el modelo reducido de una estructura, se necesita disponer:

1. De materiales y métodos adecuados que permitan la fabricación del modelo.
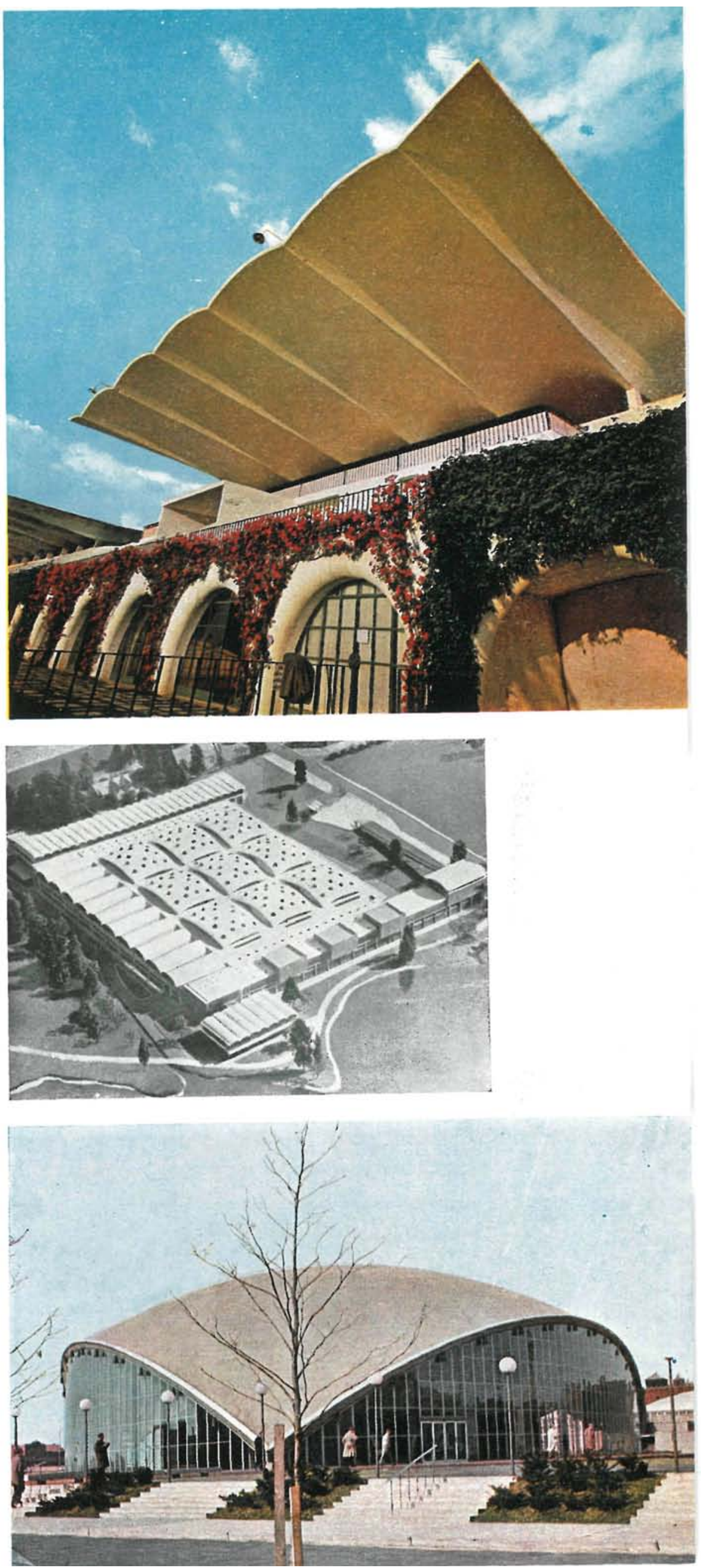

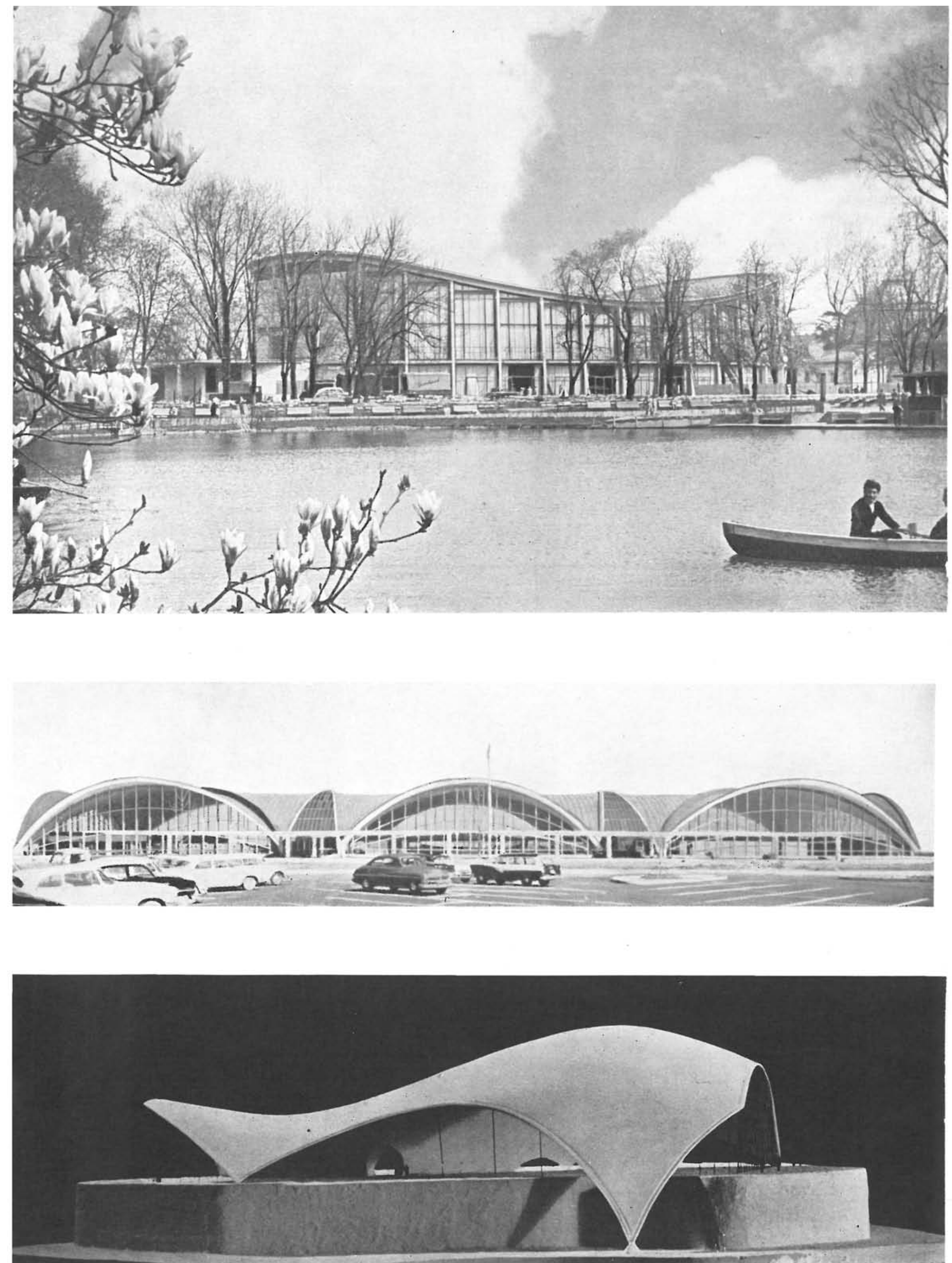


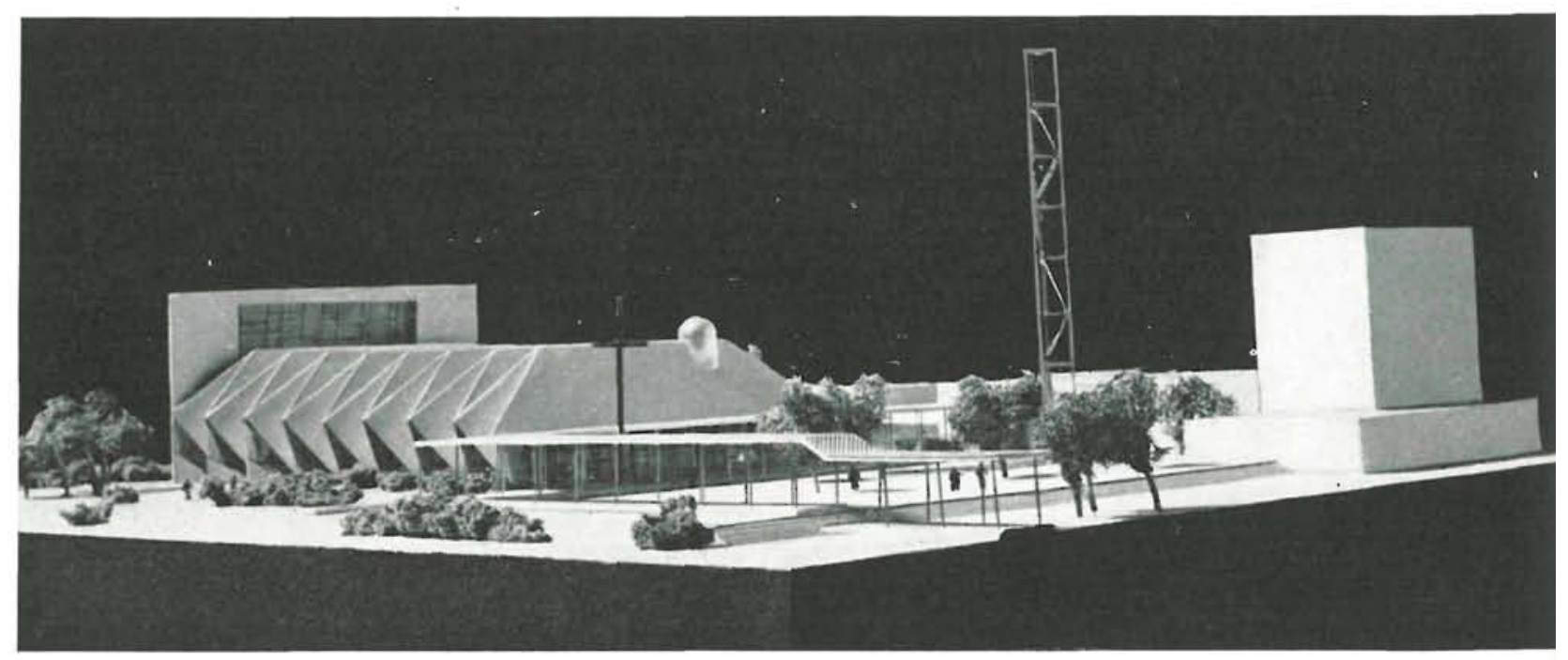

2. ${ }^{\circ}$ De procedimientos para someter el modelo a cargas análogas a las que han de actuar en la estructura real.

3. De dispositivos para medir con suficiente precisión los corrimientos, giros u otras magnitudes que puedan presentar interés en la obra real.

Se ve, por tanto, la necesidad de relacionar los resultados experimentales obtenidos del modelo con los de la estructura proyectada, para lo cual es necesario que haya una correlación, no sólo entre dimensiones, sino también entre cargas y entre características de los materiales previstos para la obra real y el modelo. De esta manera, unos resultados experimentales podrán ser trasladados a la estructura real cuyo modelo reducido se estudia. Esta relación entre modelo y estructura real es la que se establece por medio del Análisis Dimensional.
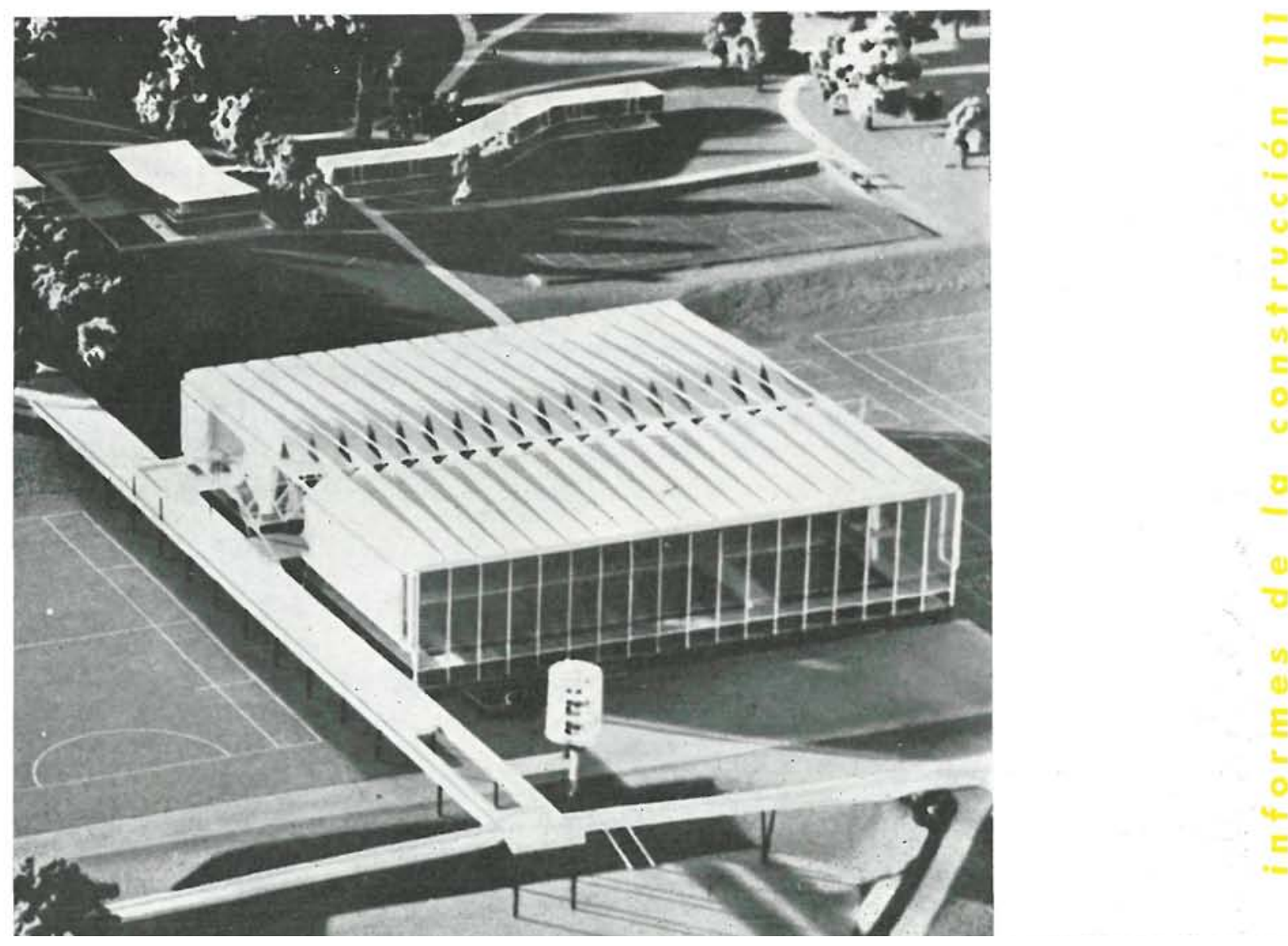
No parece oportuno introducir aquí los fundamentos del Análisis Dimensional, pues figuran en multitud de libros dedicados a ese objeto; y la aplicación concretamente del teorema $\pi$, en el caso de modelos reducidos de estructuras, figura en la publicación «Student Publications The School of Design. North Carolina State College. Raleig N. C. Volume 6 number 3».

Del citado teorema se deducen las siguientes relaciones de semejanza entre magnitudes del modelo y de la estructura real:

$$
\begin{gathered}
\frac{\sigma_{\mathrm{M}}}{\sigma_{\mathrm{R}}}=\frac{\mathrm{E}_{\mathrm{M}}}{\mathrm{E}_{\mathrm{R}}} \times \frac{\varepsilon_{\mathrm{M}}}{\varepsilon_{\mathrm{R}}}, \frac{\sigma_{\mathrm{M}}}{\sigma_{\mathrm{R}}}=\frac{\mathrm{P}_{\mathrm{M}}}{\mathrm{P}_{\mathrm{R}}} \quad " \quad \frac{\varepsilon_{\mathrm{M}}}{\varepsilon_{\mathrm{R}}}=\frac{\mathrm{C}_{\mathrm{M}}}{\mathrm{C}_{\mathrm{R}}}: \frac{\mathrm{L}_{\mathrm{M}}}{\mathrm{L}_{\mathrm{R}}} \\
\frac{\mathrm{F} \mathrm{M}}{\mathrm{F}_{\mathrm{R}}}=\frac{\sigma_{\mathrm{M}}}{\sigma_{\mathrm{R}}}\left(\frac{\mathrm{L}_{\mathrm{M}}}{\mathrm{L}_{\mathrm{R}}}\right)^{2} \quad, \quad \nu_{\mathrm{M}}=\nu_{\mathrm{R}}
\end{gathered}
$$

En dichas relaciones se designan por:

$\sigma$ las tensiones.

E los módulos de elasticidad.

$\epsilon$ las deformaciones.

$\mathrm{P}$ las presiones exteriores.

C los corrimientos.

L las longitudes.

F las cargas concentradas.

$v$ los coeficientes de Poisson.

Todos estos símbolos vienen afectados de un subíndice $\mathrm{M}$ o $\mathrm{R}$, según se trate de magnitudes del modelo o de la estructura real.

A excepción de la última relación, que expresa la igualdad en los coeficientes de Poisson del modelo o de la estructura real, no es posible sacar conclusiones sin que el problema esté determinado; pero, como en las fórmulas se manejan ocho magnitudes distintas, unidas por cinco relaciones, se ve la necesidad de fijar tres de estas magnitudes como datos del problema.

Corrientemente se conocen todas las características relacionadas con la obra real. Por tanto, son conocidos: el módulo de elasticidad, el coeficiente de Poisson y el peso específico del material, las dimensiones de la estructura y las características de las cargas, ya sean repartidas o concentradas. Hemos de fijar, por tanto, tres magnitudes del modelo para que éste quede definido.

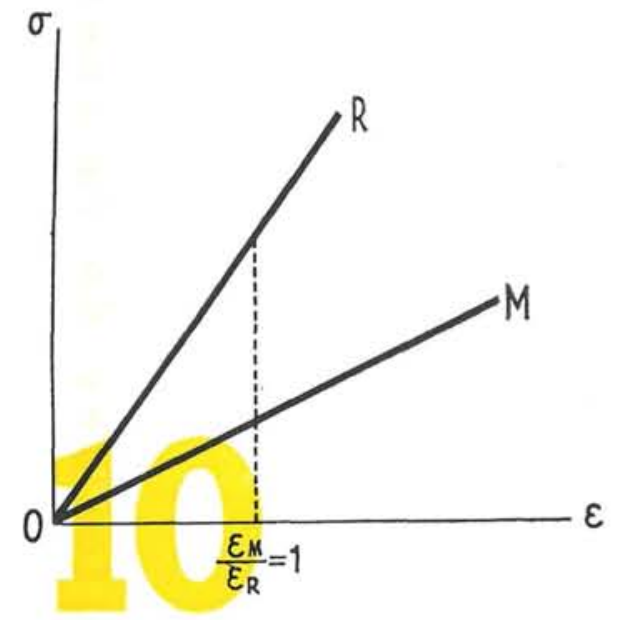

Normalmente, al proyectar el modelo se fija su tamaño, se elige un material (cuyos módulo de elasticidad, E, y coeficiente de Poisson, $v$, son conocidos) y el procedimiento de carga apropiado. De esta manera quedan conocidas las escalas de longitudes, de módulos de elasticidad y de fuerzas, con lo que pueden deducirse las demás escalas de las relaciones anteriores.

Por tanto, para que la semejanza quede definida es necesario decidir el tamaño del modelo, elegir un material y un procedimiento de cargas.

\section{Elección del tamaño del modelo}

En los ensayos con modelos reducidos conviene que éstos resulten lo más pequeños posible, dentro de unos límites admisibles, pues así se ahorran materiales en su construcción, las cargas necesarias son menores, se 
ocupa poco sitio, y el tiempo necesario para realizar la experimentación es, en general, más corto.

Las causas que limitan el grado de pequeñez del modelo son, por una parte, la imposibilidad de construir determinadas zonas de éste; y, por otra, que al reducir la escala de longitudes disminuyen simultáneamente las deformaciones relativas originadas por las cargas y, por lo tanto, se complican las operaciones de medida si han de obtenerse con suficiente precisión.

\section{Elección del material a ufilizar en la construcción del modelo}

Como se verá posteriormente, cuando se describan las aplicaciones realizadas por nosotros, para fabricar un modelo reducido pueden utilizarse diversos materiales, cuyas características resistentes son totalmente distintas. La elección debe hacerse pensando principalmente en los fines que se desean sacar de los ensayos. Si lo que se pretende es comprobar una determinada teoría (primer problema de los dos que nos hemos propuesto en el capítulo anterior), el material debe cumplir las hipótesis admitidas en esa teoría.

Por ejemplo, en el proyecto de cubiertas laminares de hormigón armado se utilizan teorías que admiten, entre otras simplificaciones, que el material de la cubierta es homogéneo, elástico e isótropo. Si se desea conocer la validez de nuevas simplificaciones en el cálculo o en las condiciones de contorno de la cubierta, es necesario que el material del modelo por estudiar sea homogéneo, elástico e isótropo para, de esta manera, comprobar la validez de las simplificaciones en el cálculo, sin que se vean interferidos los resultados por las características del material del modelo.

Según se acaba de indicar, al comprobar la validez de una teoría que parta de la hipótesis de que el cuerpo es elástico, será necesario que el material se comporte también elásticamente. Esto equivale a admitir una relación lineal entre tensiones y deformaciones para el material del modelo y de la obra real. Si con relaciones tensionales como las que se indican en la figura 10, se establece una relación entre puntos de una y otra recta, que estén en la misma vertical, entonces la escala de deformaciones resulta igual a la unidad y la escala de corrimientos, por tanto, igual a la de longitudes. De esta manera se obtiene lo que suele denominarse semejanza estricta en deformaciones, $\mathrm{y}$ todas las deformaciones producidas en el modelo por carga de peso propio o cargas exteriores producen cambios de forma semejantes a los de la obra real $\left({ }^{\circ}\right)$.

$\mathrm{Si}$, como se indica en la figura 11, se consideran como análogos puntos correspondientes a distintas verticales, la escala de deformaciones será distinta de la unidad, la escala de corrimientos no resultará igual a la de longitudes, y obtendremos lo que se suele denominar semejanza ampliada en deformaciones, igualmente válida que la anterior en la teoría de modelos, siempre que se consideren los siguientes efectos:

a) Los corrimientos originados en el modelo para unas cargas son mayores que los que se hubiesen producido en otro modelo en el cual la escala de deformaciones fuese igual a la unidad. Por tanto, cargas análogas en la estructura real y en el modelo producen, proporcionalmente, mayores corri-

(*) En este caso, las escalas de tensiones y de cargas por unidad de superficie resultan iguales a la escala de móculos de elasticidad.

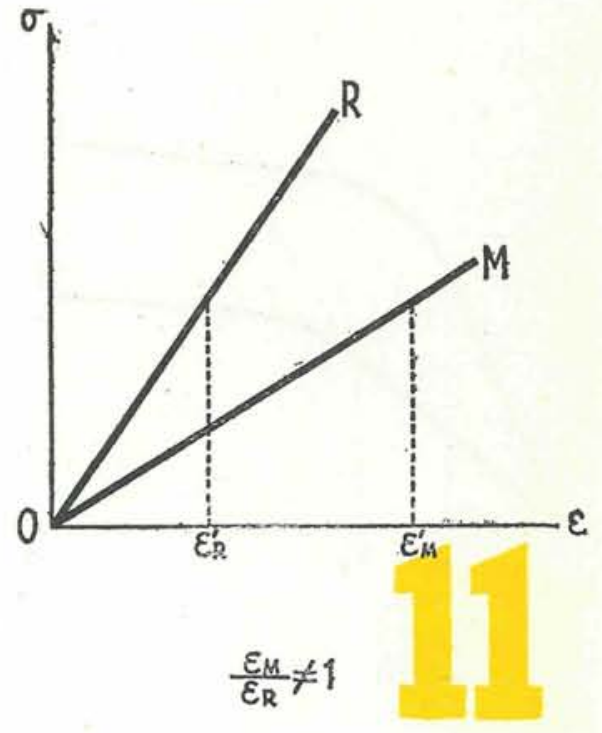




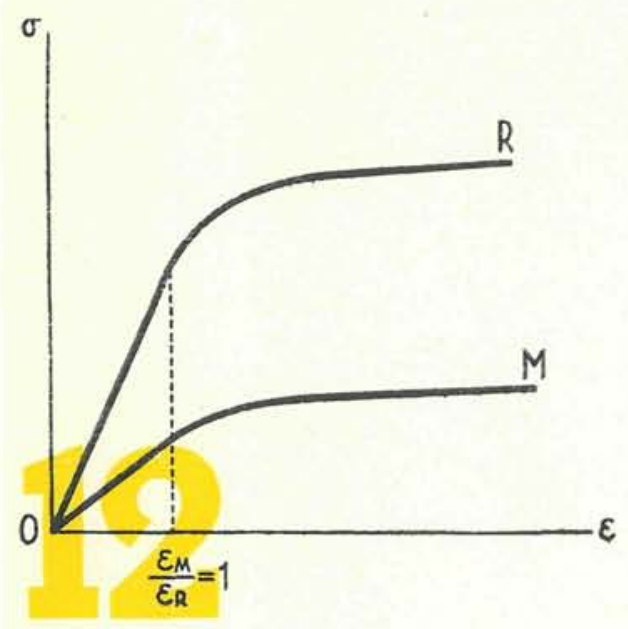

mientos en éste que en aquélla. Como es normal, en todas las teorías de cálculo de láminas no se tiene en cuenta el cambio de posición de las fuerzas, consecuencia de los corrimientos que ellas producen en la estructura. Esta hipótesis, dados los pequeños corrimientos que se producen con los materiales empleados en construcción y, principalmente, en hormigón armado, es perfectamente admisible; sin embargo, si nos imagináramos una estructura con un módulo de elasticidad muchas veces menor y con grandes corrimientos, no se puede fijar hasta qué punto sería admisible aceptar teorías que supongan pequeñas las deformaciones. Este es el efecto que produce en el modelo la semejanza ampliada que, por el motivo expuesto, no debe pasar de ciertos límites, característicos de la forma de la estructura ensayada.

b) Al manejar materiales en que el módulo de elasticidad es relativamente pequeño, disminuye considerablemente la carga crítica de pandeo de la cubierta. Por tanto, al estudiar cubiertas en régimen elástico con modelos muy deformables pueden presentarse fenómenos de inestabilidad (pandeo) que falsearían los resultados.

Cuando las relaciones entre tensiones y deformaciones de los materiales del modelo y de la obra real no son lineales, pueden presentarse dos casos de semejanza totalmente análogos a los anteriormente mencionados. En la figura 12 se recogen los diagramas tensiones-deformaciones de los materiales de la estructura y del modelo en el caso de semejanza estricta, mientras que en la figura 13 se presentan dos diagramas en los que hay que establecer la semejanza ampliada.

En el capítulo anterior planteábamos un segundo problema para resolver con los modelos, que consistía en prever, gracias a ellos, el comportamientc resistente de una estructura proyectada. Para resolverlo, es indispensable que el material del modelo y de la obra real tengan iguales características. Por tanto, si se ha proyectado una cubierta de hormigón armado, el material del modelo deberá ser anisótropo y compuesto por unas armaduras y un mortero cuyos módulos de elasticidad estén en la misma proporción que los del acero y el hormigón. En los ensayos realizados por nosotros en el Laboratorio Central de Madrid, y que se describen en el capítulo IV, hemos utilizado, en la construcción del modelo, armaduras de acero y mortero de cemento y arena, logrando un material de iguales módulo de elasticidad, coeficiente de Poisson y resistencias de rotura a tracción y compresión que los que se obtendrían a edades iguales con los hormigones de las obras reales. De esta forma, es posible estudiar con el modelo, no sólo el comportamiento elástico o cuasi-elástico de la estructura, sino también la fisuración y el colapso de la misma, lo que permite determinar el coeficiente de seguridad a rotura de la estructura real, valor éste, el más representativo, para expresar la bondad de la misma.

De acuerdo con lo hasta aquí expuesto, vamos a clasificar los modelos reducidos de cubiertas laminares en dos grupos: modelos elásticos y modelos en rotura. En el primero se reúnen todos aquellos construídos con materiales que se comportan elásticamente y en los cuales se estudian los fenómenos resistentes en período elástico. Entre los segundos incluiremos aqueİlos otros que, comportándose elástico o visco-elásticamente, como en la estructura real, es posible estudiar el comportamiento del modelo hasta el momento que se produce la rotura. 
Los materiales y los procedimientos de fabricación y de carga suelen ser distintos en ambos grupos; por tanto, se tratan a continuación en capítulos separados.

\section{III. modelos elásticos}

En este capítulo se incluye la fabricación y ensayo de modelos reducidos construídos con materiales que cumplen con las hipótesis admitidas para desarrollar la teoría de Elasticidad. Por tanto, su finalidad principal es la de servir de comprobación experimental de teorías o métodos de cálculo de láminas basados en las citadas hipótesis.

Los materiales con que se construyen estos modelos han de ser homogéneos, elásticos e isótropos, poseer una apreciable resistencia al someterlos a tracción o compresión y ser fácilmente moldeables o mecanizables para poder adaptarlos a las diversas formas previstas por el proyectista.

Con los materiales que cumplen las anteriores condiciones vamos a formar dos grupos, según sea el valor de su módulo de elasticidad. En el primero incluímos los materiales cuyo módulo de elasticidad es mayor de 15.000 $\mathrm{kg} / \mathrm{cm}^{2}$; y en el segundo, los demás. Esta distinción está originada porque los procesos de fabricación son muy distintos, como también son diferentes los procedimientos de carga y de medida que conviene utilizar y que se describirán seguidamente. Con el fin de distinguirlos, a los primeros se les denomina materiales de alto módulo de elasticidad; y los segundos, de bajo módulo de elasticidad.

\section{Modelos con materiales de alto módulo de elasticidad}

Se reúnen con esta denominación los fabricados con plexiglás, baquelita, trolón, araldita, alkateno, etc. Estos materiales se encuentran en el comercio bajo la denominación de «plásticos»: algunos, en forma de placas de diversos espesores; $\mathrm{y}$ otros, en polvo.

La fabricación de modelos con ellos debe ser realizada por personal muy especializado, y no está exenta de dificultades que conviene considerar. $\mathrm{Si}$ el material se adquiere en placas o láminas, éstas suelen ser de espesor constante; por tanto, al moldear por presión y temperatura una cubierta de doble curvatura no es fácil obtener la ley de espesores prevista por el proyectista, aunque, en muchos casos, esta dificultad puede no tener excesiva importancia. Sin embargo, antes de empezar la experimentación en un modelo de este tipo convendrá tomar, con suficiente precisión, la ley de espesores del modelo. Esta dificultad puede evitarse utilizando un material moldeable por vertido y endurecido por un tratamiento térmico, tales como la araldita y el alkateno. Con ellos pueden obtenerse modelos de la forma y espesores deseados, pero se ha de tener una especial precaución en la fabricación para que no queden tensiones internas en el modelo como consecuencia de la retracción apreciable que en ambos materiales se origina durante el endurecimiento. Este fenómeno tendrá tanta mayor importancia cuanto más hiperestática sea la cubierta.

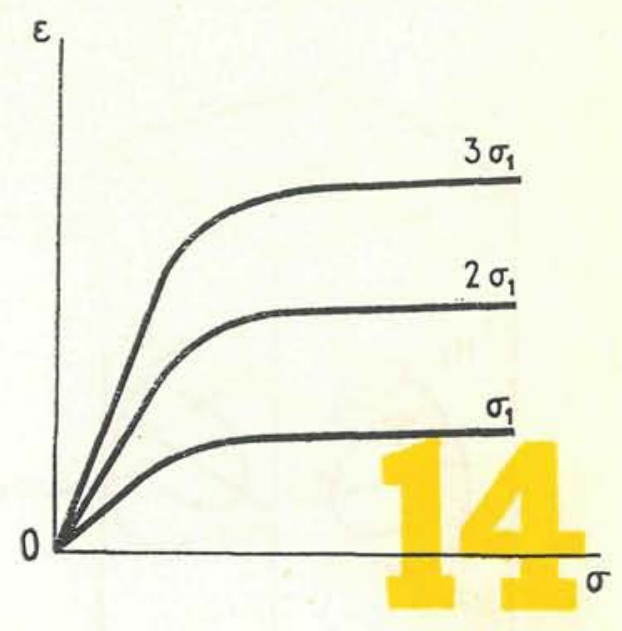




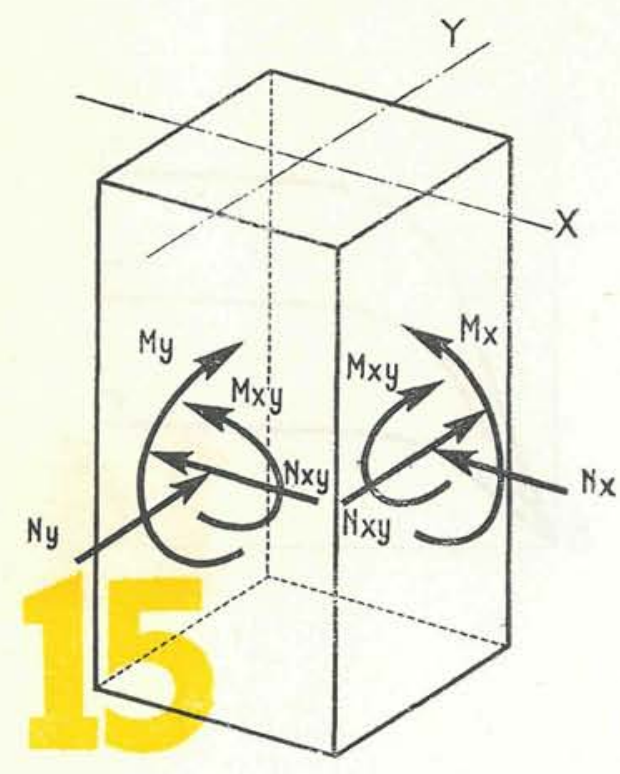

En todos estos materiales, incluídos bajo el calificativo de «plásticos», al ser sometidos a una tensión $\sigma_{1}$ se produce una deformación instantánea, que va aumentando a lo largo del tiempo, hasta alcanzar un valor prácticamente constante; y si la tensión es múltiplo de la anterior, la deformación a tiempos iguales también lo es (fig. 14). En definitiva, las deformaciones resultan proporcionales a las tensiones y el material se comporta como si cumpliese con la ley de Hooke. Conviene no ignorar esta propiedad al determinar el valor del módulo de elasticidad que se ha de utilizar en las relaciones de semejanza.

\section{Procedimiento de carga}

En las cubiertas laminares de hormigón armado o pretensado, el mayor porcentaje de carga que ha de resistir la cubierta, normalmente, corresponde a su peso propio; la nieve o el viento pueden influir de manera preponderante, según las características climáticas del lugar donde esté situada la obra; pero aún así, los efectos que obran sobre la cubierta - suma de peso propio, nieve y viento-equivalen a los producidos por cargas repartidas.

En los modelos, este tipo de cargas repartidas se suelen aplicar, siguiendo dos procedimientos: uno, colocando pesos directamente sobre la lámina, y el otro, colgándolos de la misma. El primer método permite mejor repartición de las cargas que el segundo; pero utilizándolo queda oculta la superficie superior de la estructura y, consecuentemente, surgen dificultades para la colocación de aparatos de medida sobre las superficies cargadas del modelo.

Si la sobrecarga se suspende de la estructura resistente, estas dificultades desaparecen, las superficies del modelo pueden observarse libremente y se pueden colocar los aparatos; y, si los pesos están colgados a poca altura sobre el suelo, al producirse la rotura, la cubierta se descarga casi automáticamente, permitiendo estudiar el colapso detalladamente. Al colgar la carga de la estructura resistente es necesario repartirla en gran número de pequeños pesos, que no reproducen exactamente la condición supuesta; pero si el número de éstos es muy grande, es admisible la semejanza entre la carga repartida y la constituída por un numeroso conjunto de cargas concentradas de igual valor total.

El método de colgar un gran número de contrapesos por debajo de la cubierta requiere una condición que, de olvidarse, hace que los resultados de la experimentación se presenten enmascarados. Esta condición exige, manifiestamente, que la carga se comporte durante los ensayos como lo haría al actuar sobre la estructura real, es decir, si se estudia el efecto del peso propio, nieve o viento, actuando según una ley prefijada, sobre toda la cubierta del modelo, todas las cargas deben colocarse simultáneamente, ya que si se cuelgan pesos para lograr las cargas, éstos no deben suspenderse intermitentemente, colocando unos ahora, otros después, porque se correría el riesgo de crear estados intermedios de carga más peligrosos que los totales. Esta dificultad se soslaya en parte, dejando las partes inferiores de todos los contrapesos apoyadas en un mismo tablero, al que se hace descender lentamente cuando se quiere actuar las cargas sobre el modelo. 


\section{Dispositivos de medida}

En este tipo de modelos se pueden medir corrimientos con una precisión de $0,01 \mathrm{~mm}$ en direcciones cualesquiera, aunque corrientemente se haga según las normales a la superficie media de la lámina; giros, por medio de clinómetros, con una sensibilidad de 0,0001 radiantes, y deformaciones en las superficies de la cubierta, por medio de extensómetros mecánicos o eléctricos (strain-gage). Con estos últimos es posible precisar deformaciones de $1 \times 10^{-5}$ con una base de medida de $10 \mathrm{~mm}$.

\section{Cálculo de esfuerzos}

En las teorías utilizadas para calcular estructuras laminares, se define como cuña elemental, en un punto, según dos ejes cartesianos $\mathrm{X}$ e $\mathrm{Y}$, tangentes a la superficie media de la lámina en sus dos radios de curvatura principales, a la que resulta de cortarla, primero, por dos planos infinitamente próximos según la dirección del eje $\mathrm{X}$ y, después, por otros dos planos infinitamente próximos, según la dirección del eje Y. Todos ellos deben ser perpendiculares a la superficie media de la lámina y contener los dos radios de curvatura principales, correspondientes al punto en que se cortan.

En el caso más general, el equilibrio de la cuña elemental requiere que en sus superficies laterales actúen unos momentos de flexión y de torsión y unos esfuerzos normales y tangenciales como los indicados en la figura 15.

Los valores de estos esfuerzos pueden deducirse de los resultados de las medidas de las deformaciones en las superficies del modelo por el procedimiento que a continuación se indica: Supóngase que en el modelo de una cubierta laminar, representado parcialmente en la figura 16, se desean conocer los esfuerzos que, como consecuencia de las cargas, actúan en las superficies laterales de la cuña elemental, HJKL-H'J'K'L', cuya posición y orientación se conoce. Para definir la posición de la cuña elemental, se trazan los ejes rectangulares $\mathrm{X}$ e $\mathrm{Y}$, definidos anteriormente.

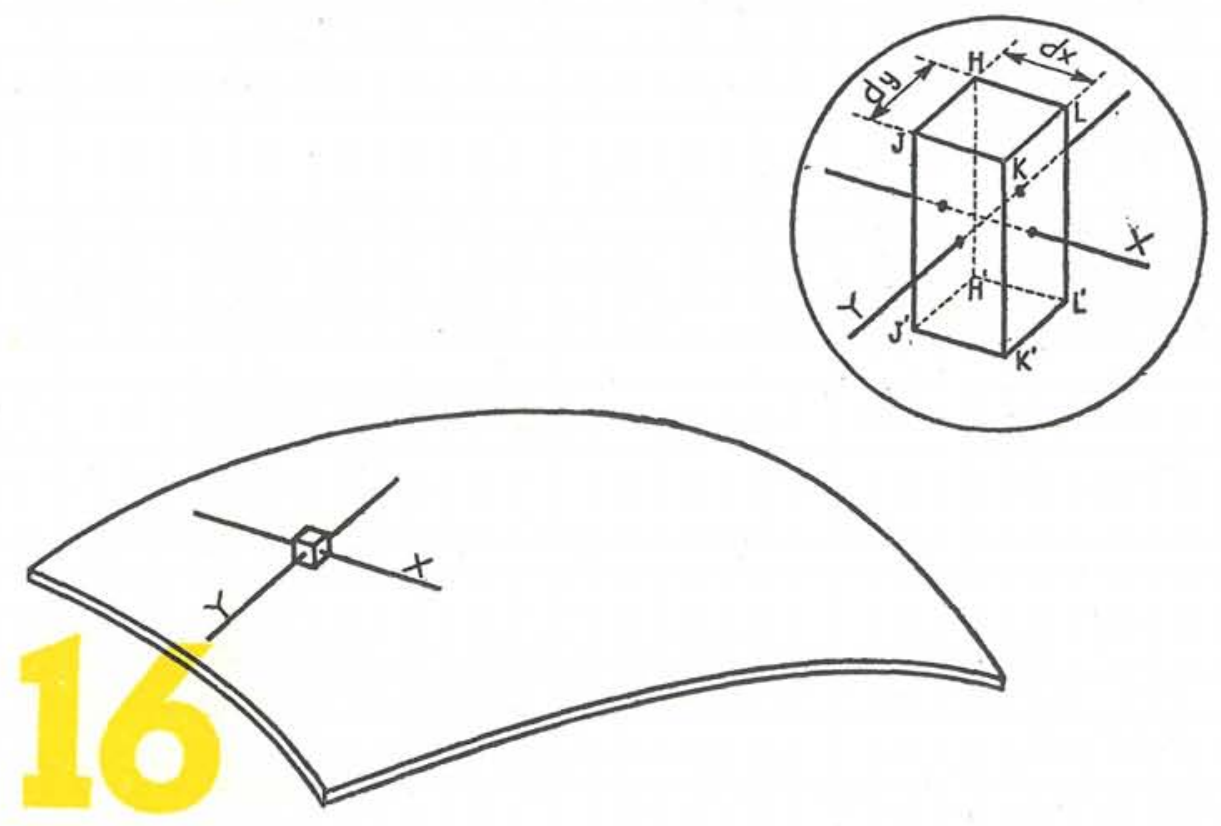




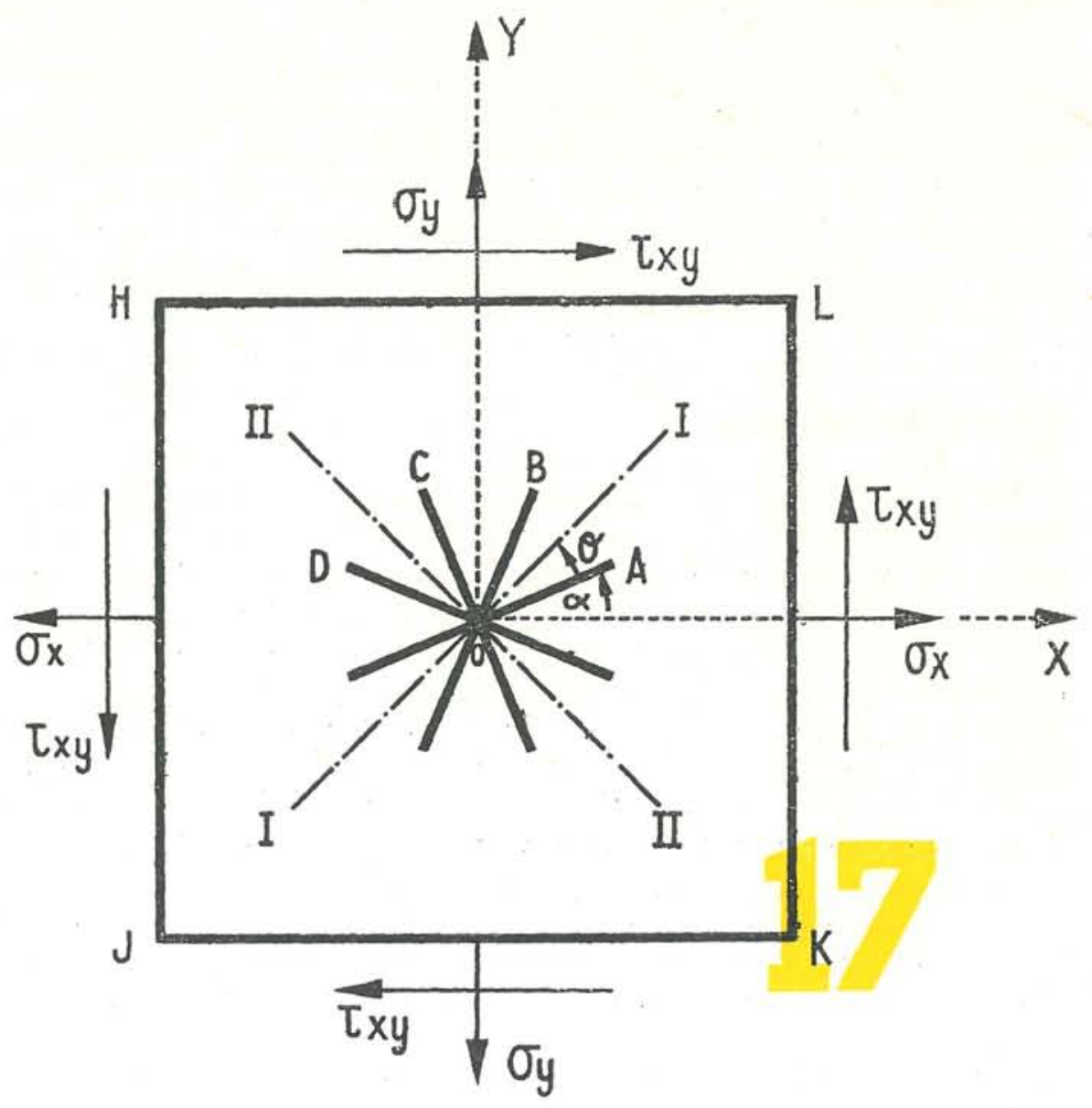

En cada una de las bases de la cuña elemental así definida se pegan cuatro elongámetros eléctricos cruzados en roseta equiangular $\left({ }^{\circ}\right)$. La orientación de la roseta puede ser arbitraria, pero siempre es conocido el ángulo que forma con el eje $\mathrm{X}$ uno cualquiera de los extensómetros. Al someter el modelo reducido de la estructura a las cargas previstas, se conocerán las deformaciones según cuatro direcciones en cada base de la cuña elemental. Así, en la base superior HJKL (fig. 17) son conocidos los valores de $\alpha$ y de $\varepsilon_{A}, \varepsilon_{B}, \varepsilon_{C}$ y $\varepsilon_{D} ;$ si los resultados experimentales fuesen exactos, tendría que verificarse que

$$
\varepsilon_{\mathrm{A}}+\varepsilon_{\mathrm{C}}=\varepsilon_{\mathrm{B}}+\varepsilon_{\mathrm{D}}
$$

pero como esto ocurre raras veces, se determinan los valores y direcciones de las deformaciones y tensiones principales por medio de las siguientes expresiones, deducidas aplicando la teoría de los mínimos cuadrados $\left({ }^{\circ}{ }^{\circ}\right)$ :

$$
\begin{aligned}
& \varepsilon_{\mathrm{I}}=\frac{1}{4}\left[\varepsilon_{\mathrm{A}}+\varepsilon_{\mathrm{B}}+\varepsilon_{\mathrm{C}}+\varepsilon_{\mathrm{D}}+2 \sqrt{\left(\varepsilon_{\mathrm{A}}-\varepsilon_{\mathrm{C}}\right)^{2}+\left(\varepsilon_{\mathrm{B}}-\varepsilon_{\mathrm{D}}\right)^{2}}\right] \\
& \varepsilon_{\mathrm{II}}=\frac{1}{4}\left[\varepsilon_{\mathrm{A}}+\varepsilon_{\mathrm{B}}+\varepsilon_{\mathrm{C}}+\varepsilon_{\mathrm{D}}-2 \sqrt{\left(\varepsilon_{\mathrm{A}}-\varepsilon_{\mathrm{C}}\right)^{2}+\left(\varepsilon_{\mathrm{B}}-\varepsilon_{\mathrm{D}}\right)^{2}}\right]
\end{aligned}
$$

(*) La determinación de las deformaciones principales, sólo requiere tres extensómetros; pero, en la práctica, se colocan cuatro para poder compensar la influencia de pequefios errores experimentales. Una forma de colocarlos es la roseta equiangular, que es aquella en la que los
cuatro extensómetros se cruzan, de manera que cada eje longitudinal forme ángulos de $45^{\circ}$ con los contiguos.

(**) "Determination of Principal Stress from Strains of Four Intersecting Gage Lines $45^{\circ}$ Apartn, por William R. Osgood. Journal of Research of the National Bureau of Standards, vol. 15, december 1935, pág. 580 . 


$$
\begin{gathered}
\sigma_{\mathrm{I}}=\frac{E}{1-\nu^{2}}\left(\varepsilon_{\mathrm{I}}+\nu \varepsilon_{\mathrm{II}}\right) ; \\
\sigma_{\mathrm{II}}=\frac{E}{1-\nu^{2}}\left(\varepsilon_{\mathrm{II}}+\nu \varepsilon_{\mathrm{I}}\right) ; \\
\operatorname{tg} 2 \theta=\frac{\varepsilon_{\mathrm{B}}-\varepsilon_{\mathrm{D}}}{\varepsilon_{\mathrm{A}}-\varepsilon_{\mathrm{C}}} .
\end{gathered}
$$

En estas expresiones se denomina $\sigma_{I}$ a la tensión principal de mayor valor algebraico; $\mathrm{y} \varepsilon_{\mathrm{I}}$, a la deformación según esa dirección. $\theta$ es el ángulo que forma la dirección de $\sigma_{\mathrm{I}}$ con la dirección del elongámetro $\mathrm{OA}$, que es conocida. Conocidos los valores de $\sigma_{\mathrm{I}} ; \sigma_{\mathrm{II}} \mathrm{y} \alpha+\theta$, que es el ángulo que forma $\sigma_{I}$ con el eje $\mathrm{X}$, el cálculo de $\sigma_{x}, \sigma_{y}$ y $\tau_{x y}$ puede hacerse por las fórmulas clásicas de la Elasticidad plana.

Por el procedimiento expuesto, y partiendo de unos resultados experimentales, pueden calcularse las tensiones $\sigma_{x}, \sigma_{y} \tau_{x y}, \sigma_{x}^{\prime}, \sigma_{y}^{\prime}$ y $\tau_{x y}^{\prime}$ en los extremos superior e inferior de las superficies laterales de la cuña elemental (fig. 18). Si se admite la hipótesis de variación lineal de tensiones según el espesor de la lámina, la determinación de los valores de los momentos de flexión y de torsión y de los esfuerzos normales y tangenciales, puede hacerse por medio de las siguientes expresiones:

$$
\begin{aligned}
& \mathrm{M}_{x}=\frac{c^{2}}{12}\left(\sigma_{x}^{\prime}-\sigma_{x}\right) ; \mathrm{M}_{y}=\frac{c^{2}}{12}\left(\sigma_{y}^{\prime}-\sigma_{y}\right) ; \mathrm{M}_{x y}=\frac{c^{2}}{12}\left(\tau_{x y}^{\prime}-\tau_{x y}\right) ; \\
& \mathrm{N}_{x}=\frac{c}{2}\left(\sigma_{x}^{\prime}+\sigma_{x}\right) ; \mathrm{N}_{y}=\frac{c}{2}\left(\sigma_{y}^{\prime}+\sigma_{y}\right) ; \mathrm{N}_{x y}=\frac{c}{2}\left(\tau_{x y}^{\prime}+\tau_{x y}\right) ;
\end{aligned}
$$

en donde $c$ representa el espesor de la lámina.

Se ve, que de los resultados experimentales obtenidos al ensayarse un modelo reducido de una cubierta laminar pueden deducirse los valores de corrimientos, giros o esfuerzos en diversas secciones del modelo o de la estructura real, supuesta construída con un material homogéneo y elástico. Esto es lo admitido por muchas teorías; por tanto, los resultados obtenidos en la experimentación son muy útiles para comprobar la validez de determinados métodos de cálculo, que, admitiendo las citadas hipótesis, no hayan sido sancionados por la experiencia.

Además, como estos procedimientos dan resultados basados en hipótesis admitidas corrientemente, cabría la posibilidad para el proyectista creador de una forma original, cuyo cálculo fuese muy complejo o inabordable, de hacer un primer dimensionado basado en formas de trabajo sencillas. Ensayar un modelo elástico, y con los valores de los esfuerzos deducidos experimentalmente mejorar, si fuese preciso, la forma o las dimensiones y pasar, por último, al ensayo de un nuevo modelo elástico o en rotura. De este último tipo se tratará posteriormente.

\section{Modelos con materiales de bajo módulo de elasticidad}

Los modelos cuya fabricación y ensayo acaba de mencionarse ofrecen grandes ventajas, pero presentan el grave inconveniente de su difícil fabricación y el de necesitar unos dispositivos de carga muchas veces complicados

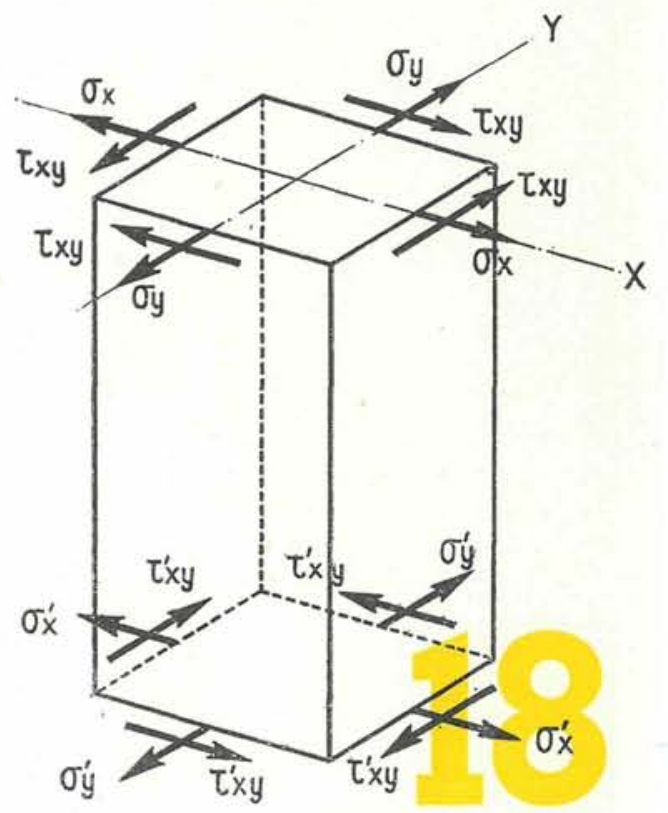


en exceso. Con el fin de disminuir al máximo los dispositivos de carga de los modelos, se nos ocurrió la construcción de éstos con materiales suficientemente deformables, para que, actuando únicamente el peso propio del mismo modelo, se produjesen corrimientos, deformaciones y giros, medibles con suficiente aproximación.

Como el peso propio actúa sobre el modelo al quitar los moldes o encofrados, es muy difícil colocar los aparatos para medir deformaciones en cualquier punto de la superficie del modelo antes que empiece a sentirse este efecto. La dificultad puede vencerse dejando que actúe el peso del modelo para colocar luego los aparatos de medida. Después se giran el modelo y los soportes de los aparatos $180^{\circ}$ alrededor de un eje horizontal. Este procedimiento tiene las dos ventajas siguientes:

1. ${ }^{a}$ Permite repetir el proceso de carga todas las veces que sea preciso.

2. ${ }^{a} \quad$ Las magnitudes medidas son el doble de las que corresponderían a una carga igual al peso propio, lo que equivale a duplicar la sensibilidad del sistema de medida (1).

El material utilizado por nosotros para esta clase de modelos es el que denominamos «litargel», compuesto por una mezcla de litargirio, gelatina, glicerina y agua. Modificando convenientemente las proporciones entre los citados componentes, pueden obtenerse materiales de visco-elástico y cuyos módulos de elasticidad pueden variar a voluntad entre 10 y $500 \mathrm{~kg} / \mathrm{cm}^{2}$.
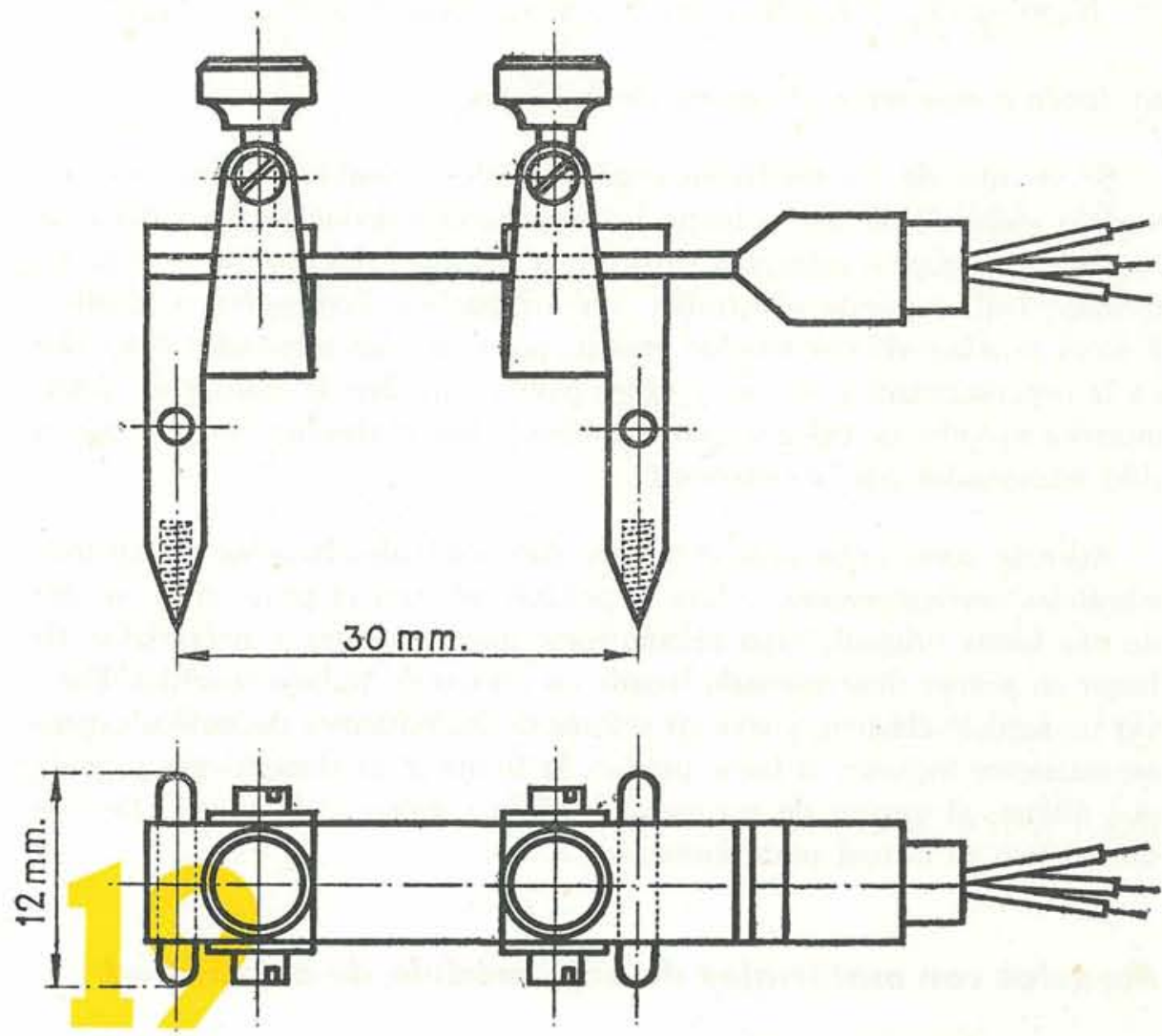

(1) Este procedimiento no puede aplicarse en aquellos casos en que al girar el modelo alrededor de un eje horizontal, se originen estados de carga que ocasionen la rotura de aquél. 
Hasta el momento presente, estos materiales presentan el inconveniente de tener un coeficiente de Poisson algo más alto que el del hormigón, pues suele estar comprendido entre 0,3 y 0,4, y tanto más próximo a este último valor cuanto más bajo es el módulo de elasticidad.

Este material, preparado a $50^{\circ} \mathrm{C}$ tiene el aspecto de un líquido algo viscoso con el que pueden fabricarse los modelos de la forma y dimensiones que se deseen, inyectándole en moldes construídos con yeso, cuyas superficies pueden prepararse con pintura celulósica para evitar la adherencia entre el material del modelo y el molde. Al enfriarse el material y alcanzar la temperatura ambiente, la solución de gelatina se convierte en un gel muy deformable, pero que va aumentando la rigidez al paso del tiempo, según reacciona el litargirio con la glicerina. Después de unos cinco a siete días, según las mezclas, el modelo queda preparado para el ensayo.

\section{Dispositivos de medida}

Con estos materiales tan deformables que queremos sean suficientemente sensibles solamente por su peso propio, es necesario disponer de unos procedimientos de medida que, a su gran precisión, unan el tener muy poco peso. En principio se pensó utilizar elongámetros eléctricos que, como es sabido, se aplican pegados sobre la superficie del modelo por intermedio de una tira de papel, pero nos encontramos con la grave dificultad de que la rigidez del papel era suficiente para falsear el ensayo. Desechados los elongámetros mecánicos de que disponemos, pues tienen un peso excesivo, hemos construído unos, en los que dos elongámetros eléctricos van pegados a la viga de un pórtico cuyas patas se apoyan sobre la superficie del modelo. Cada aparato, cuya forma y dimensiones se indican en la figura 19, pesa sólo 9 gramos, lo que representa una carga concentrada, cuyo equivalente en la construcción real es prácticamente despreciable. Su rigidez, por otra parte, es sumamente pequeña y no influye en los resultados del ensayo, como hemos podido comprobar con probetas sometidas a compresión simple.

Con el fin de no perder precisión al deducir las tensiones principales, lo ideal sería conocer las isostáticas antes de colocar los aparatos, para, de esta manera, en vez de cuatro elongámetros, colocar solamente dos, según las direcciones de las tensiones principales. Con este material esto es posible; pues si se aplica sobre sus dos superficies una capa muy fina de laca frágil, al hacer que actúe el peso propio la laca se fisura dibujando las isostáticas de tracción; y éstas son fácilmente visibles pulverizando sobre el modelo una lluvia muy fina de sulfuro de sodio, la cual, al penetrar por las pequeñas grietas, ataca al óxido de plomo del litargel y forma sulfuro de plomo de color negro. En la descripción de la parte experimental realizada se incluyen fotografías y dibujos de las isostáticas obtenidas.

\section{Cálculo de esfuerzos}

Conocidas las direcciones de las deformaciones principales en las superficies de las láminas, por medio de lacas frágiles pueden determinarse experimentalmente los valores de las deformaciones principales en las bases de un prisma elemental; y a partir de estos datos, el cálculo de los esfuerzos se hace de manera idéntica a la expuesta en este capítulo para los modelos construídos con materiales de alto módulo de elasticidad. 


\section{Resumen del méfodo}

Consiste en construir el modelo reducido de la cubierta laminar, con un material homogéneo, que resista a tracción y compresión, y cuyo módulo de elasticidad sea suficientemente pequeño para que sólo el peso propio del modelo produzca deformaciones, corrimientos y giros que se puedan medir con precisión.

\section{Aplicaciones}

Para tener una idea de los órdenes de magnitud de las diferentes cantidades que intervienen en el problema, vamos a suponer que se desea construir el modelo reducido de una lámina de hormigón armado con unas determinadas dimensiones en planta, y un espesor medio de $8 \mathrm{~cm}$, y la cual va a estar sometida, entre su peso propio y la sobrecarga que sobre ella pueda actuar, a una carga de $320 \mathrm{~kg} / \mathrm{cm}^{2}$. Si por conveniencias de tamaño construímos el modelo a escala $1 / 10$, el espesor medio del modelo será de $8 \mathrm{~mm}$; y si el peso específico del material empleado es de 2 tons $/ \mathrm{m}^{3}$, el modelo pesará $16 \mathrm{~kg} / \mathrm{m}$. Por tanto, la escala de presiones o cargas por metro cuadrado será de $16 \times 2 / 320=1 / 10$, que es la misma escala en que estarán relacionadas las tensiones del modelo y de la obra real.

Es necesario tener en cuenta que al estar el modelo constituído por un material homogéneo, las tensiones máximas en sus superficies son menores que las análogas obtenidas en un modelo igual construído con mortero armado. Pues si una sección de hormigón armado o de mortero armado con una cuantía de $1 \%$, como es lo corriente, tiene un diagrama de tensiones semejante al indicado en la figura 20 a, con sección homogénea se obtendría el de la figura $20 \mathrm{~b}$, en el que las tensiones máximas son aproximadamente el $60 \%$ de las originadas en el caso anterior. Por tanto, si admitimos una carga máxima en el hormigón de la lámina real a compresión de $50 \mathrm{~kg} / \mathrm{cm}^{2}$, en una lámina de las mismas dimensiones, pero construída con material homogéneo, la máxima tensión sería, aproximadamente, de $0,6 \times 50=30$ kilogramos $/ \mathrm{cm}^{2}$, y la máxima tracción o compresión en el modelo sería de $3 \mathrm{~kg} / \mathrm{cm}^{2}$.

Supongamos ahora que queremos precisar las tensiones en el modelo con un error menor de 1/100 de la tensión máxima; ello equivaldría, en la realidad, a obtener una precisión de $0,5 \mathrm{~kg} / \mathrm{cm}^{2}$, que estimamos como muy buena. Con nuestros extensómetros se pueden precisar deformaciones de $1 \times 10^{-4} \mathrm{y}$, por tanto, el módulo de elasticidad del material resultaría de $0,03 / 10^{-4}=300 \mathrm{~kg} / \mathrm{cm}^{2}$. Es decir, si se construye el modelo con un material que tenga este módulo de elasticidad, que se comporte como material elástico, tanto a tracción como a compresión con tensiones menores de $3 \mathrm{~kg} / \mathrm{cm}^{2}$, y que tenga un peso específico de $2 \mathrm{tons} / \mathrm{m}^{3}$, su peso propio, solamente, producirá en él un efecto análogo al que en la estructura real produciría el peso propio de esta estructura más la sobrecarga prevista; y las medidas se obtendrían, en la realidad, con una precisión de $0,5 \mathrm{~kg} / \mathrm{cm}^{2}$. 


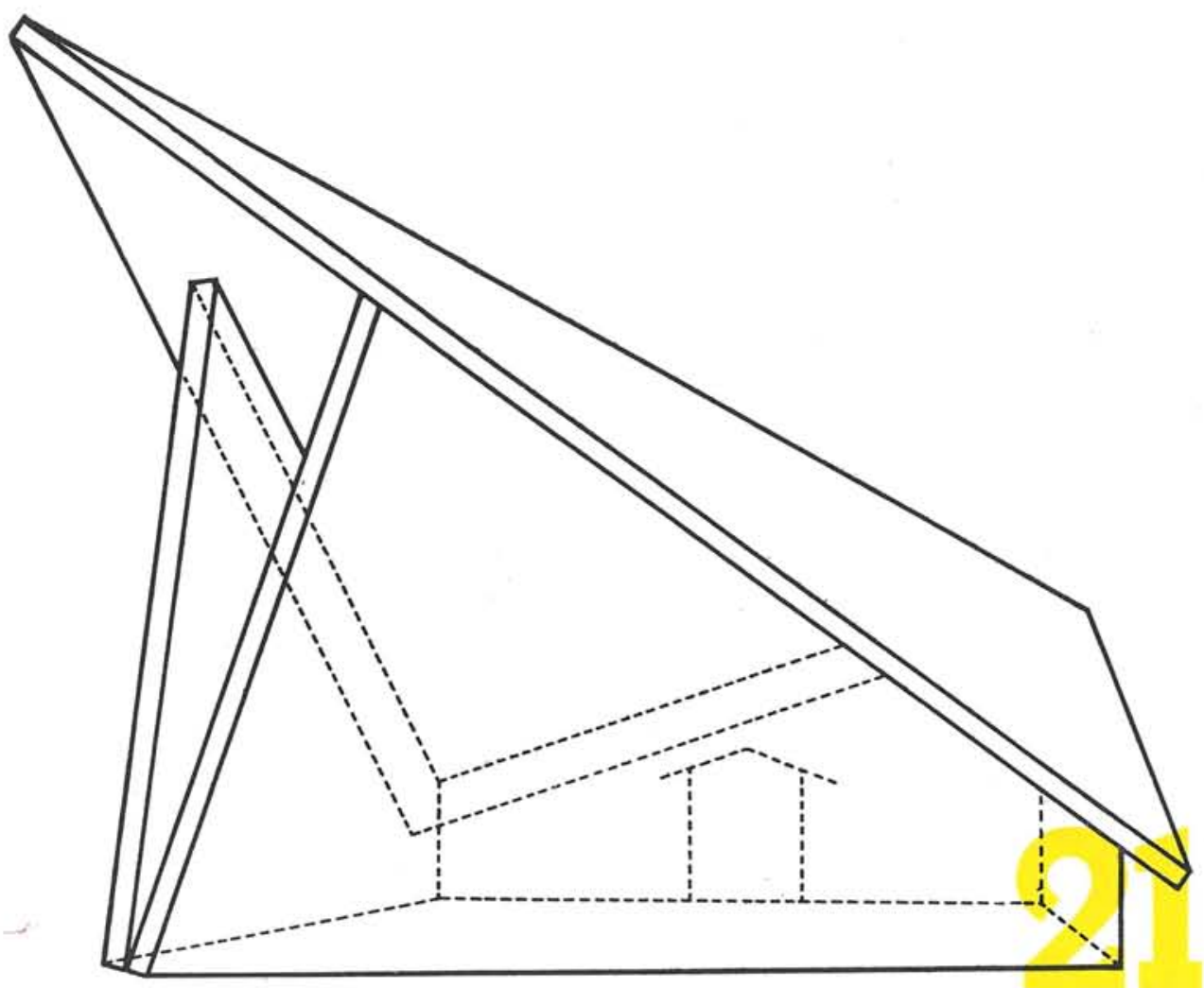

\section{Experiencias realizadas}

El profesor Torroja, en colaboración con el arquitecto señor Rodríguez Mijares, ha proyectado una capilla que ha sido construída en el Alto Pirineo español. Su forma en planta es un cuadrilátero cuyas diagonales miden 19 y 13 metros, respectivamente. Su cubierta está formada por dos placas iguales, de hormigón armado, cada una de las cuales tiene forma de triángulo rectángulo. Ambas placas están empotradas entre sí por sus hipotenusas. La cubierta se apoya sobre los muros, que no forman recinto cerrado, pues una de las esquinas ha sido sustituída por un amplio ventanal, delante del cual está situado el altar. Una perspectiva del conjunto puede verse en la figura 21.

El peso propio de la cubierta y las sobrecargas de viento y nieve producen empujes inclinados sobre los muros del contorno; y, aunque éstos pueden suponerse rígidos en las tres esquinas sólidas, se deforman en las proximidades del ventanal produciendo flexiones secundarias en la cubierta. En los ensayos realizados se deseaba conocer el estado de tensiones, suponiendo indeformables los muros. Para ello se ha construído el modelo de la cubierta a escala $1 / 15$ (fig. 22), y se han sustituído los muros de apoyo por una estructura metálica que sujeta al contorno de la cubierta, por debajo y por encima, permitiendo los giros de la lámina, y de tal forma, que la sustentación no varía al dar el modelo una vuelta de campana, según el procedimiento de carga anteriormente descrito.

Después de moldeado el modelo se aplicó, sobre sus superficies exterior e interior, una capa de laca frágil y, de esta manera, al actuar el peso propio se obtuvieron las isostáticas en casi toda la superficie (fig. 23). Se observó 


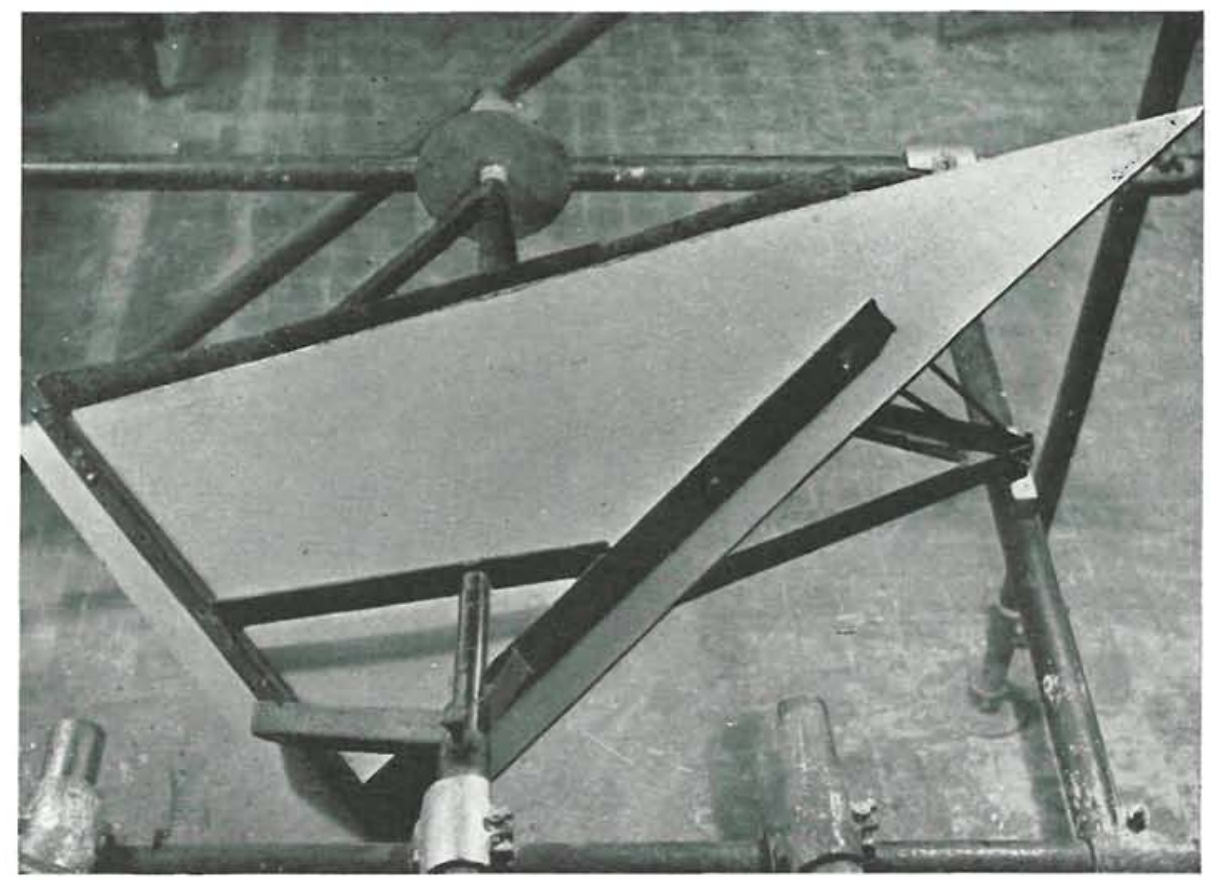

22

que en pequeñas zonas no se producían fisuras en la laca, debido a que los valores de las tensiones en las superficies eran muy pequeños. Con estos datos y algunas medidas complementarias, se han dibujado las isostáticas representadas en la figura 24 . Las isostáticas, representadas en la mitad superior de dicha figura, corresponden a las de la superficie exterior de la cubierta; y las otras, a las de la superficie interior. Del estudio comparativo de las inclinaciones de las isostáticas en las superficies exterior e interior de un paralelepípedo elemental, se deduce de manera cualitativa la importancia de los esfuerzos cortantes y de torsión.

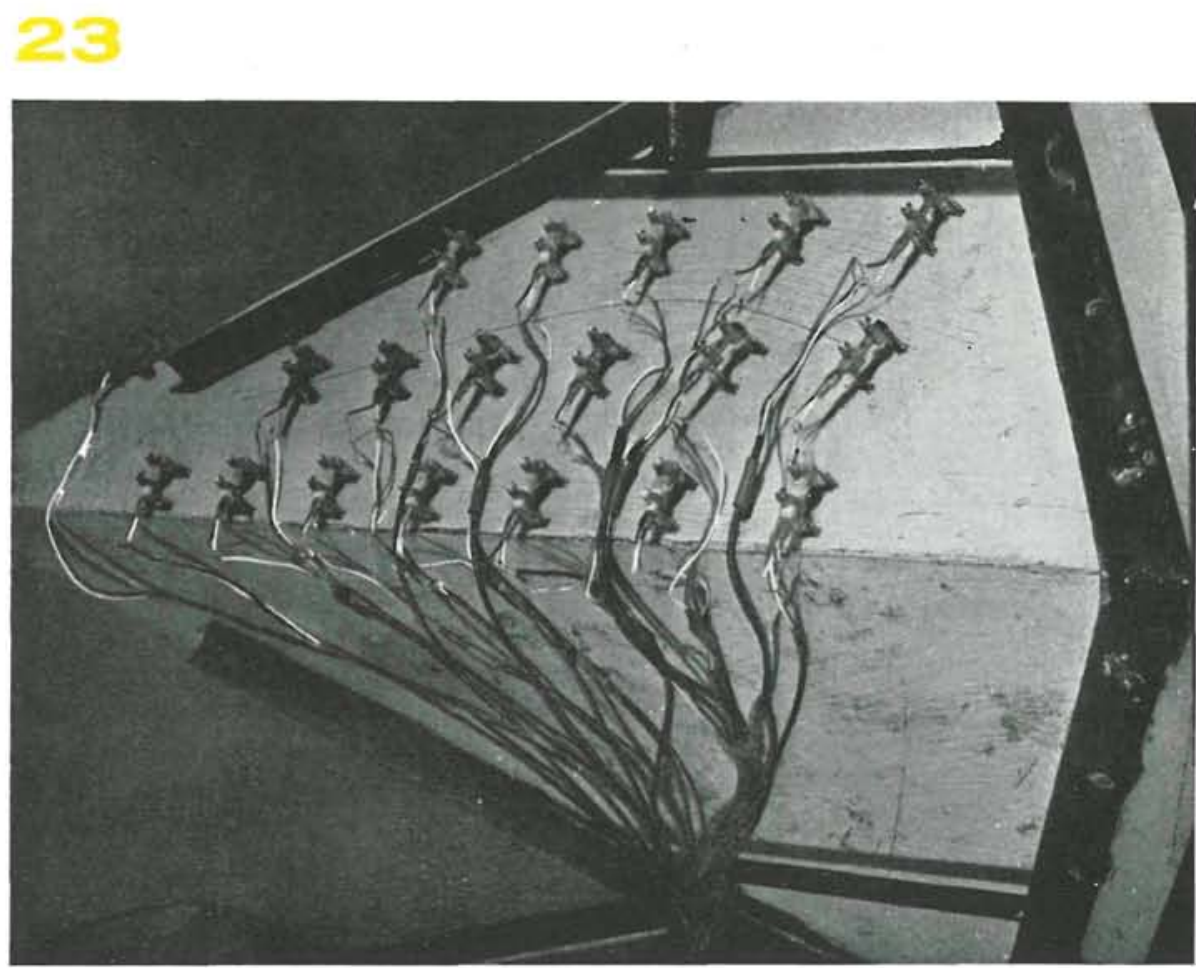



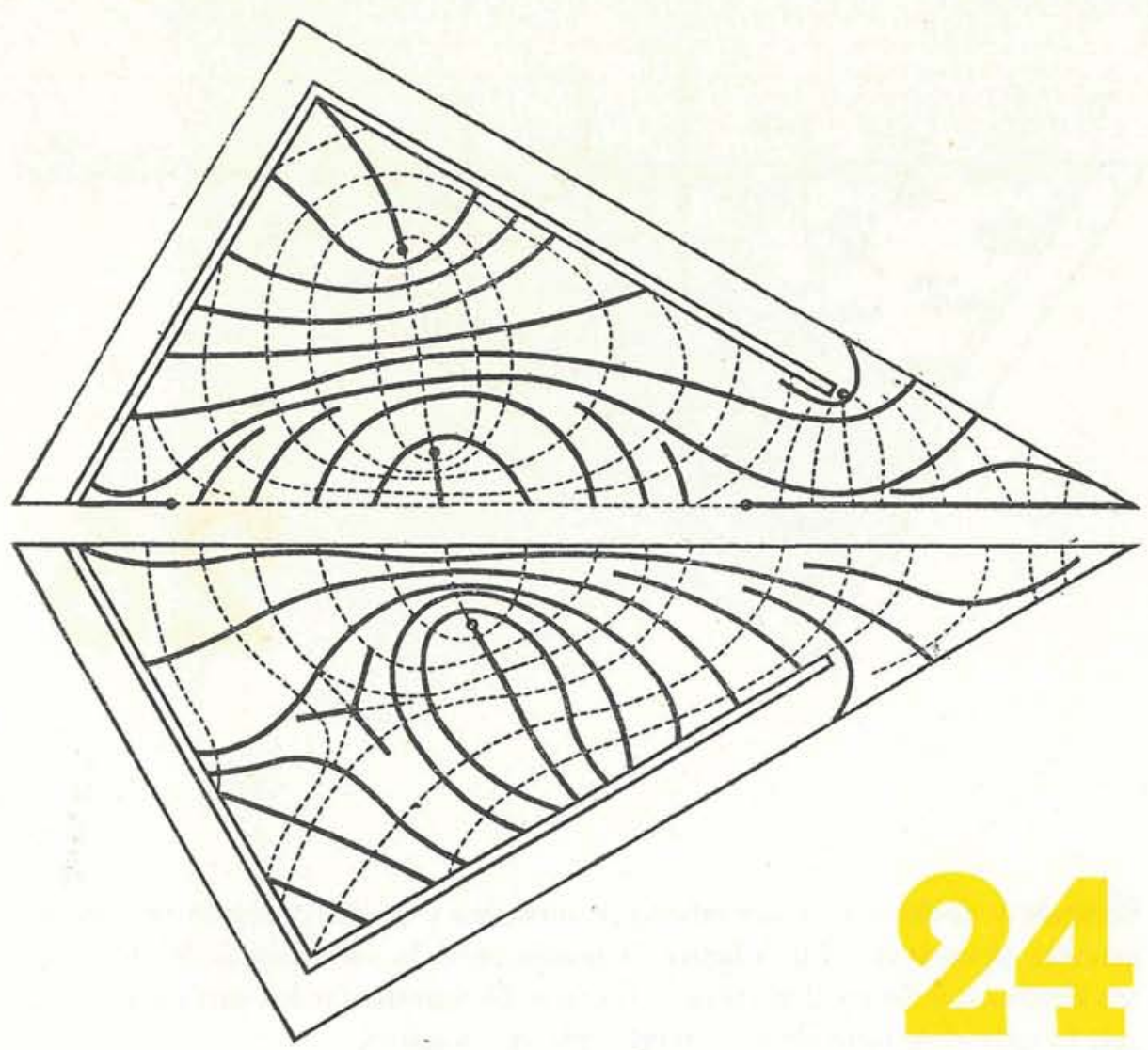

Una vez obtenidas las direcciones de las deformaciones principales en las superficies, se colocaron elongámetros eléctricos en varios puntos y se determinaron los valores de las referidas deformaciones. Después, y aplicando las condiciones de semejanza del análisis dimensional, pudieron hallarse los valores de las tensiones principales con un error menor de $0,5 \mathrm{~kg} / \mathrm{cm}^{2}$ en las superficies de una cubierta real construída con materiales homogéneos. Estos resultados se indican en las figuras 25 y 26 . A partir de ellos, y por el método indicado anteriormente, se calcularon los momentos

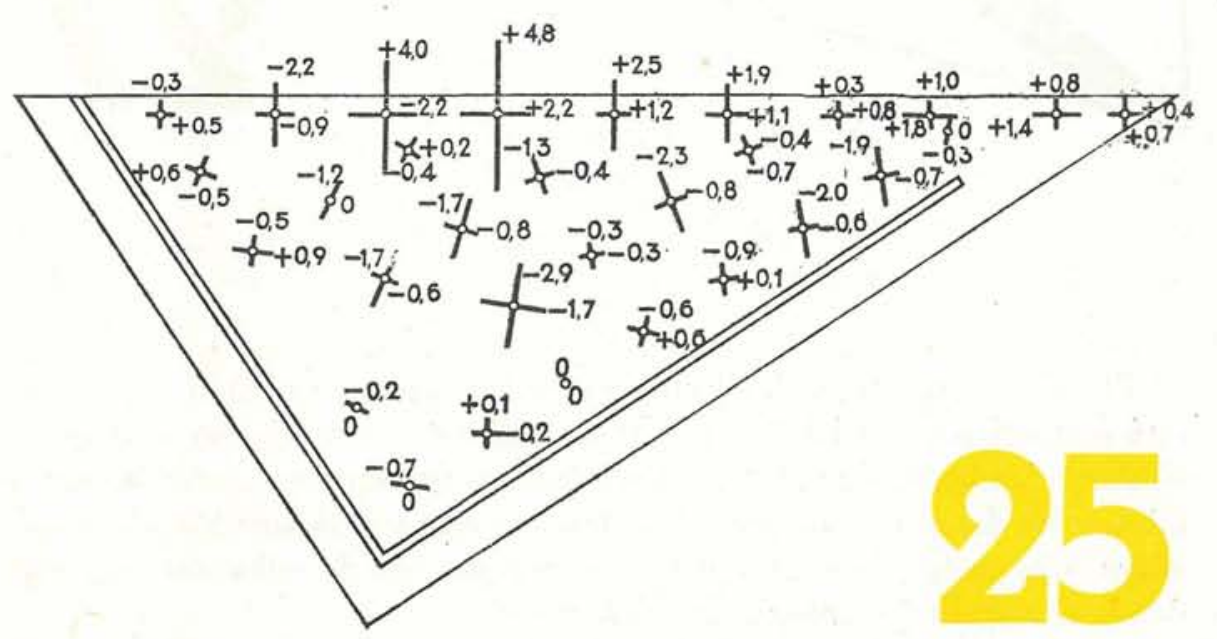




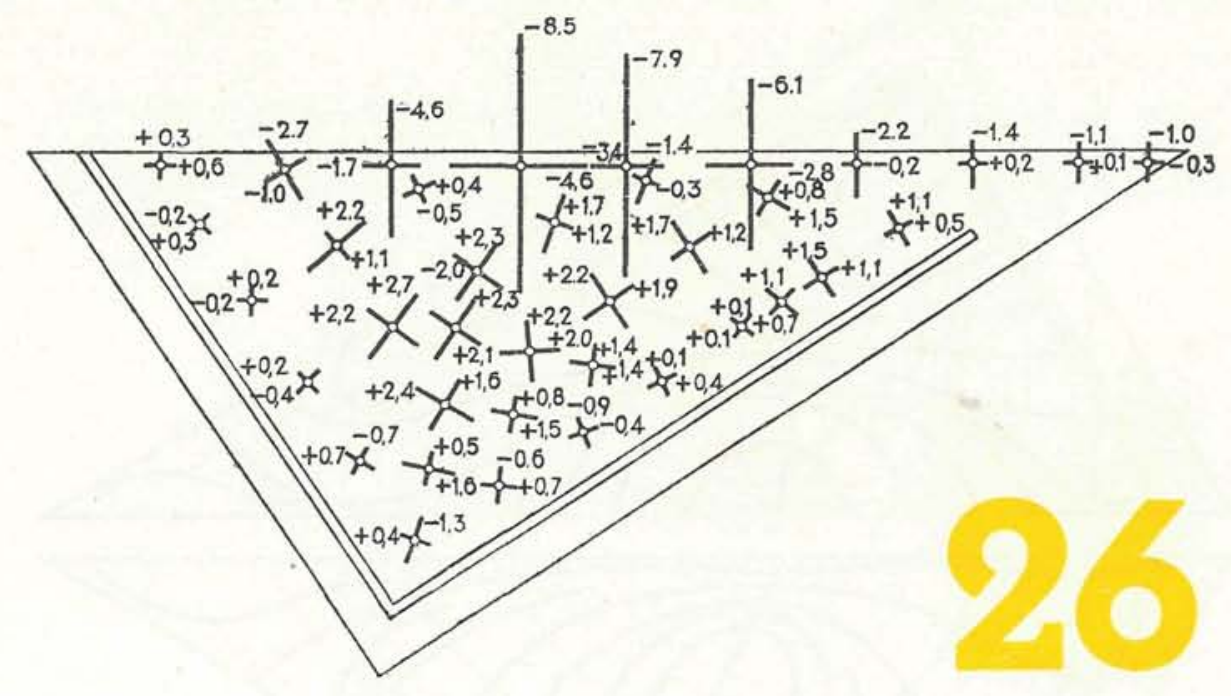

flectores y de torsión, y los esfuerzos normales y tangenciales en veintiocho prismas elementales. En la figura 27 puede verse la variación de los momentos flectores; y de igual manera, sería posible representar los otros esfuerzos, con lo que el estudio de la cubierta queda completo.

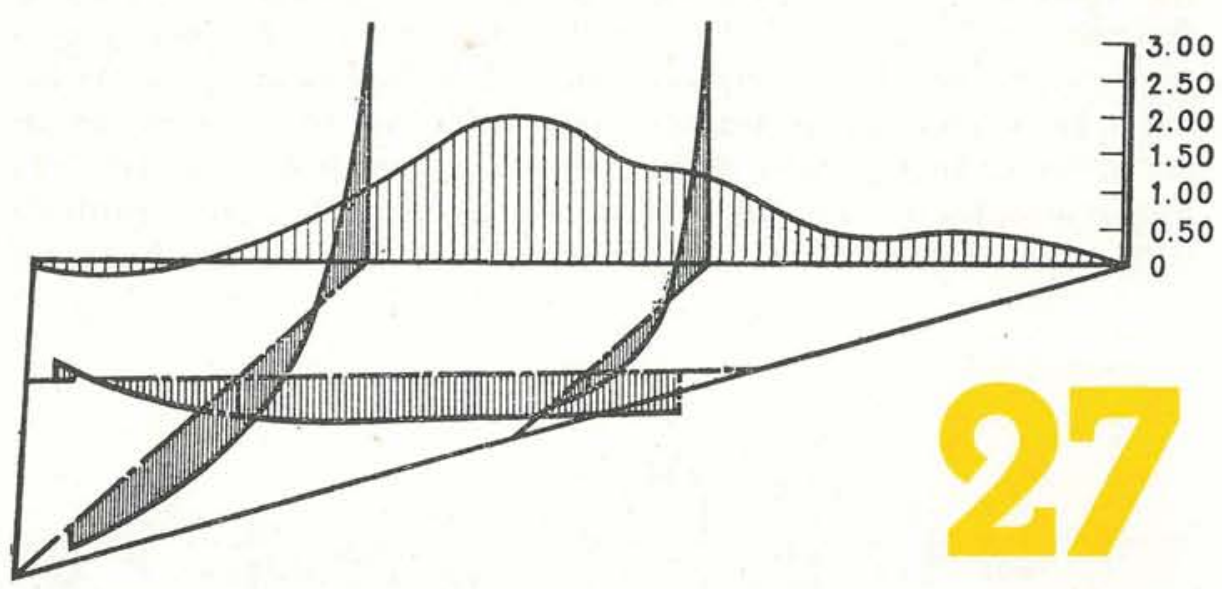

El método, no sólo es aplicable en este ejemplo, en el cual la cubierta está formada por dos láminas planas de espesor constante, sino también en cubiertas laminares de una o dos curvaturas y de espesor variable. Como la fabricación del modelo, el procedimiento de carga y el de medida son económicos, este método resulta útil para cualquier tipo de cubiertas laminares donde el cálculo presente dificultades. 